\title{
DEL POPULISMO AL PROGRESISMO: reflexiones sobre su capacidad transformadora ${ }^{1}$ DO POPULISMO AO PROGRESSISMO: reflexões sobre sua capacidade de transformação
}

\author{
Blanca Rubio* \\ Jaime Peña* *
}

\begin{abstract}
El objetivo del artículo consiste en analizar y contrastar el populismo de los años 30 con el progresismo de los años 2000 en América Latina, poniendo énfasis en el ámbito rural. Se trata de indagar cuáles fueron las condiciones que permitieron el potencial transformador del populismo y cuáles aquellas que generaron el fenómeno reciente, que castraron su capacidad de cambio. El argumento principal apunta a las condiciones históricas en las que surgieron ambos fenómenos. Mientras el populismo surgió cuando ya existían las condiciones para sustituir a la oligarquía terrateniente por la burguesía industrial e impulsar el régimen de acumulación por sustitución de importaciones, el progresismo emerge prematuramente, cuando se ha iniciado el declive del régimen neoliberal pero aún no se ha debilitado el poder del capital financiero y corporativo, ni se vislumbra la sustitución de dicha clase por otro sector. Esto lleva a una incapacidad del progresismo para impulsar cambios estructurales.

Palabras clave: Transición Hegemónica. Populismo. Progresismo. Hegemonía.

O objetivo do artigo é analisar e contrastar o populismo dos anos 1930 com o progressivíssimo dos anos 2000 na América Latina, com ênfase no meio rural. Trata-se de investigar quais foram as condições que permitiram o potencial transformador do populismo e quais foram as que geraram o fenômeno recente, que castrou sua capacidade transformadora. $\mathrm{O}$ argumento principal aponta para as condições históricas em que ambos os fenômenos surgiram. Enquanto o populismo surgiu quando já existiam as condições para substituir a oligarquia latifundiária pela burguesia industrial e promover o regime de acumulação por substituição de importações, o progressivíssimo surgiu prematuramente, quando o declínio do regime neoliberal havia começado, mas ainda não se debilitou. O poder do capital financeiro e empresarial, nem a substituição dessa classe por outro setor dominante à vista. Isso leva a uma incapacidade do progressismo de promover mudanças estruturais.

Palavras chave: Transição Hegemônica. Populismo. Progressivíssimo. Hegemonia.
\end{abstract}

\section{INTRODUCCIÓN}

Uno de los fenómenos relevantes de la etapa reciente, lo constituye la emergencia de gobiernos alternativos en América Latina, que desafiaron las políticas impuestas desde los años ochenta por el Fondo Monetario Internacional y el Banco Mundial. A partir de 1999, año en que tomó el poder Hugo Chávez en Venezuela, se replicaron triunfos semejantes

\footnotetext{
* Universidad Nacional Autónoma de México. Instituto de Investigaciones Sociales.

Circuito, Mario de La Cueva S/N, C.U. Cep: 04510 CDMX. Coyoacán - México. blancaa@unam.mx https://orcid.org/0000-0001-7753-6573

* * Universidad Nacional Autónoma de México. Facultad de Estudios Superiores Acatlán.

Avenida Alcanfores y San Juan - Totoltepec s/n - Sta. Cruz Acatlán. Cep: 53150 Naucalpan de Juárez - México. jiper48@yahoo.com.mx

https://orcid.org/0000-0001-6215-7853

1 Una primera versión de este artículo fue presentada como ponencia en el XXXII Congreso de ALAS, celebrado en Lima Perú, del primero al 6 de diciembre de 2019. Agradecemos la participación de Jorge Tripp en la recopilación y sistematización de la información utilizada.
}

en Uruguay, Brasil, Argentina, Bolivia, Ecuador, El Salvador, Honduras, Paraguay y México. Este proceso, conocido como la "marea rosa”, inspiró un sinnúmero de estudios que han buscado tanto su significado como su capacidad transformadora, lo cual generó un intenso debate principalmente entre las visiones críticas del capitalismo. ${ }^{2}$ El ascenso del progresismo ocurre en una fase de transición del capitalismo, identificada por la decadencia del régimen de acumulación neoliberal y la crisis de la hegemonía norteamericana, procesos en los cuales se debilitaron las clases dominantes, pero sobre todo sus representaciones políticas, lo que permitió el ascenso de gobiernos disi-

${ }^{2}$ Las posiciones principales para el objeto de este trabajo son aquellas que reivindican la capacidad transformadora del progresismo como Emir Sader (2009) y Atilio Borón (2013), entre otros y aquellas que consideran que no solo no transformaron las estructuras sino que maniataron los movimientos sociales (Modonesi, 2012; Svampa, 2018a; Gudynas, 2010; Kay; Vergara, 2018), entre otros. Sobre los textos que abordan el debate véase Gudynas (2012), Svampa (2018b) y Katz, (2008). 
dentes del neoliberalismo. Dichos gobiernos surgieron impulsados por grandes movimientos populares frente al hartazgo de la exclusión social y la forma represiva de gobernar que predominó durante la fase neoliberal.

Uno de los rasgos relevantes de los gobiernos antineoliberales que ha causado mayor debate, lo constituye el bajo potencial para transformar estructuralmente a las economías e impulsar un nuevo régimen de acumulación alternativo a la vía Neoliberal. Este fenómeno contrasta claramente con el proceso conocido como populismo que emergió en el marco de la crisis de 1929 en América Latina, y constituyó una de las fuerzas más importantes para impulsar el régimen de acumulación denominado Sustitución de Importaciones, el cual trajo consigo una etapa "incluyente" de las masas populares. Al igual que el progresismo, el populismo ocurrió en una fase de transición, con la fractura del liberalismo y la decadencia de la oligarquía terrateniente, en el contexto del declive de la hegemonía británica.

En este ámbito, el objetivo del artículo consiste en analizar y contrastar el populismo de la primera mitad del siglo XX con el progresismo de los años 2000, con el fin de indagar cuáles fueron las condiciones históricas que permitieron el potencial transformador del populismo y cuáles obstaculizaron cambios estructurales en el progresismo, poniendo énfasis en el ámbito rural. Toda vez que se trata de స్․ un tema muy amplio, se darán líneas generales i debido a la limitación del espacio, tanto en las causas de los procesos como en las transformaciones rurales y las políticas públicas, sin profondizar en las especificidades de cada país.

$\dot{2} \quad$ En el primer punto se aborda la visión fi teórica y metodológica, para enseguida tratar la transición del siglo XX y el populismo, se aborda después la transición del siglo XXI y el progresismo. Al final se plantea una visión comparativa entre los dos procesos.

\section{VISIÓN TEÓRICA Y METODOLÓGICA}

El punto de partida metodológico de este ensayo lo constituye el concepto de transición hegemónica capitalista, que contiene tanto una crisis del régimen de acumulación como una crisis de hegemonía mundial. Es en el contexto del debilitamiento del poder, cuando "lo viejo no acaba de morir y lo nuevo no termina por nacer" (Gramsci, 1981, p. 58) que surgen este tipo de regímenes, que son por su naturaleza transitorios y tienden a declinar cuando se configuran las condiciones para el surgimiento y consolidación de los procesos que sustituyen a los decadentes.

Desde esta perspectiva, el presente artículo pretende aportar una visión crítica del fenómeno, desde dos visiones poco analizadas: una referida a las transformaciones rurales y otra desde un enfoque histórico estructural, que permita dilucidar las condiciones económicas que dieron pie en cada fase, a cambios de orientación política y transformaciones económicas y sociales, bajo la figura carismática de un líder con gran atracción popular. La iniciativa de analizar el populismo como referente esencial parte de la convicción de que, aun cuando se trata de períodos y fenómenos históricos diferentes, son susceptibles de comparación, pues tanto el populismo como el progresismo surgen en etapas de transición, en el marco de un vacío de poder de las elites dominantes, con el apoyo de las masas populares e inspirados por una visión antiimperialista. ${ }^{3}$

Por otra parte, el populismo aporta claves fundamentales que permiten profundizar en el conocimiento de los regímenes progresistas de la actualidad. Toda vez que se trata de un fenómeno acabado y ampliamente estudiado, se afina la visión del progresismo y surgen interrogantes de profundidad para caracterizar sus alcances. Sin embargo, es necesario te-

${ }^{3}$ Varios autores han hecho también comparaciones de los dos procesos, como Atilio Borón (2012), Enrique Dussel (2012), Adrián Piva (2013), a la vez que múltiples autores analizan el progresismo haciendo referencias puntuales del populismo, como Ugo Pipitone (2015), Carlos Vilas (1994) y Claudio Katz (2008), entre otros. 
ner precaución en este tipo de confrontaciones históricas pues como señala Carlos Vilas (2005, p. 58):

La identificación de una especie de parentesco político entre algunas dimensiones de las propuestas de cambio de la nueva izquierda y de sus estilos políticos con aspectos o dimensiones de los regímenes nacional-populares que protagonizaron importantes experiencias de democratización y transformación social en el siglo XX tiene sentido en la medida en que sea llevada a cabo con extrema cautela. Las ideas suelen sobrevivir a los escenarios que les dieron nacimiento, pero los regímenes políticos expresan siempre la impronta de los escenarios sociales en los que se desenvuelven, y deriva de ellos mucho de su identidad efectiva.

Desde un punto de vista teórico, partimos de la crítica de la economía política, por considerar que es el enfoque más potente para analizar la etapa actual. El marxismo es la corriente que ha desarrollado en mayor medida la teoría de la crisis capitalista y, por tanto, la que tiene las herramientas para entender los procesos de transición como los que analizamos.

En cuanto a los conceptos, existe un amplio debate que refiere al populismo, el cual no desarrollamos aquí, toda vez que no es el objetivo del artículo. Las posiciones más influyentes han sido aquellas como la de Ernesto Laclau para quien

“el populismo es la articulación de temas populares
en oposición al bloque en el poder. Cuando esta ar-
ticulación antagónica de los temas populares es lle-
vada a cabo por las clases explotadas, el populismo
es de izquierda, progresista; cuando es efectuada
por fracciones periféricas a las clases dominantes,
que intentan reestructurar el bloque en el poder en
beneficio propio, el populismo es reaccionario (es
decir, fascista)" (Mouzelis, 1994, p. 445).

A diferencia de esta concepción, en este artículo consideramos fundamental abordar el populismo como un fenómeno histórico y no como una categoría analítica (Moira; Petrone, 1999). Desde esta perspectiva, el populismo corresponde a una etapa específica del capitalismo y es, según Ianni (1975, p. 148), un “modo de organización política de las relacio- nes de producción en una época en la que crecen las fuerzas productivas y el mercado interno". En este sentido, el populismo constituye la expresión política de una configuración estructural determinada en ciertas sociedades de América Latina, en una etapa definida. Para Vilas (1994, p. 42):

[...] el populismo puede ser visto como un movimiento de masas que aparece en el centro de las rupturas estructurales que acompañan a la crisis del sistema capitalista mundial y a la crisis de las oligarquías latinoamericanas [...] Puede ser visto, asimismo, como la respuesta a una crisis de hegemonía (ausencia de dirección política) en el marco de una crisis del sistema capitalista y de las presiones por impulsar la industrialización por encima de los límites que fijaba el sistema exportador tradicional.

En este sentido no se puede, por tanto, llamar populismo o neopopulismo al proceso que ocurre en la etapa actual, toda vez que emerge en una etapa histórica diferente y bajo determinaciones causales distintas. Por esta razón consideramos más acertado llamarle progresismo, en tanto es el término más difundido en el debate sobre el tema. ${ }^{4}$ Según Sader (2009, p. 108) este fenómeno: ${ }^{5}$

No caracteriza una etapa histórica específica, diferente del capitalismo y del socialismo, sino una nueva configuración de las relaciones de poder entre las clases sociales, que promete la formación de un nuevo bloque social dirigente de procesos históricos suigéneris, en condiciones mucho más favorables a las fuerzas populares, cuyo destino será decidido por una dinámica concreta de construcción de Estados postneoliberales.

Desde nuestro parecer, el progresismo es un fenómeno histórico que corresponde a la transición capitalista de la hegemonía norteamericana y del régimen neoliberal, en la cual ocurre un debilitamiento del dominio del capi-

${ }^{4}$ Aun cuando es el término más utilizado existen otras visiones. Desde posiciones que le llaman "ciclo de impugnación al neoliberalismo" (Ouviña; Thwaites, 2018), o bien "revoluciones pasivas" (Modonesi,2017). Para otros autores se reivindican los conceptos como neodesarrollismo o socialdesarrollismo (Katz, 2015) entre otros.

${ }^{5}$ Emir Sader se refiere en esta cita a lo que él llama postneoliberalismo. Lo retomamos sin embargo por considerarlo válido también para el progresismo. 
tal financiero y corporativo, así como el declive del poder de Estados Unidos y los organismos multilaterales sobre los países de América Latina, que permite el ascenso de gobiernos no alineados, sostenidos sobre las masas populares, que impulsan procesos de nacionalización de los recursos naturales así como políticas redistributivas del gasto público, con una visión antiimperialista.

\section{LA TRANSICIÓN HEGEMÓNICA DEL SIGLO XX Y EL POPULISMO}

A principios del siglo XX ocurrió un largo período de transición entre el régimen de acumulación liberal y el régimen por sustitución de importaciones, a la par con el paso de la hegemonía mundial de Gran Bretaña a Estados Unidos. Se trató de una transformación de gran calado en el ámbito mundial que requirió de dos guerras mundiales y una crisis capitalista de orden estructural.

La transición inició con la crisis mundial de 1873 y entró en una crisis de hegemonía con la primera guerra mundial, conocida como "la gran guerra" y tuvo su punto más álgido en la crisis de 1929, como reflejo del agotamiento del régimen liberal de producción.

La crisis capitalista tuvo como causas estructurales el agotamiento de una forma particular de funcionamiento del régimen liخิ beral, el cual se sustentó en el estancamiento กิ de los salarios y el declive de los precios de los alimentos y las materias primas, con el fin de ¿ impulsar las ganancias, a la vez que combinó $\stackrel{10}{\rightarrow}$ elevados incrementos de la productividad del $\dot{2}$ trabajo con políticas deflacionarias.

ले El resultado de esta forma de funcionamiento en las etapas de expansión, lo constituye la emergencia de la sobreacumulación de capital y la sobreproducción de mercancías, en tanto la demanda no crece al mismo ritmo que la productividad, pues tanto los obreros como los campesinos fueron sometidos a bajos salarios y bajos precios.
Al ocurrir un sobrante de productos y de capital, sin posibilidades de realización y reinversión rentable debido a la estrechez del mercado, suele ocurrir que el capital emigre hacia el área especulativa para valorizarse.

El proceso especulativo tiende a separarse de la esfera de la producción, por lo que se crea una burbuja bursátil que estallará cuando se agoten las condiciones para su reproducción, ya sea por cambios en las políticas monetarias o bien cuando los valores se intentan vender sin que surjan compradores y van perdiendo nivel, llevando a la ruina a quienes habían invertido en ellos.

Esto fue lo que ocurrió en la crisis de 1929, cuando la separación del valor y su representación dineraria llevó al crack bursátil, al que sobrevinieron las quiebras bancarias y la reducción del crédito que afectó a las empresas, con lo cual se redujo la producción y el empleo.

En el ámbito de las materias primas, el estallamiento de la crisis trajo consigo el declive brutal de los precios:

\footnotetext{
En los meses que van de noviembre de 1929 a enero de 1939, la media del índice (de los precios y stocks agrícolas mundiales) fue de 64 , con un descenso del 9\% respecto al nivel de julio-octubre. Y después llegó el diluvio: 58 en marzo de 1930; 51.4 en junio; 45.5 en agosto, y, en diciembre de 1930, 38.9, lo que significaba una disminución del 50\% desde la primera mitad de 1928. En diciembre de 1932 el índice había disminuido casi en otro 50\%, hasta un $24.4 \%$ de la base de 1923-25, mientras que los stocks habían crecido hasta 260, medidos sobre esa misma base (Kindleberger, 2009, p. 139).

La caída en el precio de las materias primas agrícolas y mineras en el ámbito mundial fue la puntilla que acabó por debilitar a la oligarquía exportadora en América Latina. Esta clase de la burguesía nativa empezó a resentir además el declive en la demanda de bienes primarios debido a la crisis que enfrentaron las grandes potencias, por lo que se generó un vacío de poder en el ámbito de la representación política, que permitió el ascenso de líderes ajenos a las filas de la clase en el poder.
} 
En estos procesos ocurre un intenso antagonismo ya que ninguna clase puede imponerse sobre las otras, de modo que, desde una perspectiva gramsciana, las fuerzas en lucha se equilibran de una manera catastrófica a través de una coalición de antagónicos (Ianni, 1975, p. 57).

Surge así el régimen populista, como un modo de organización política de las relaciones de producción, que representa a la clase emergente; una clase que por su debilidad germinal no puede impulsar el nuevo régimen de producción y debe ser impuesto por el Estado. De esta suerte la burguesía nacional industrial coman-

Gráfica 1 - Precio Internacional del Trigo, 1900-1960 USD / Bushel $(1990=100)$

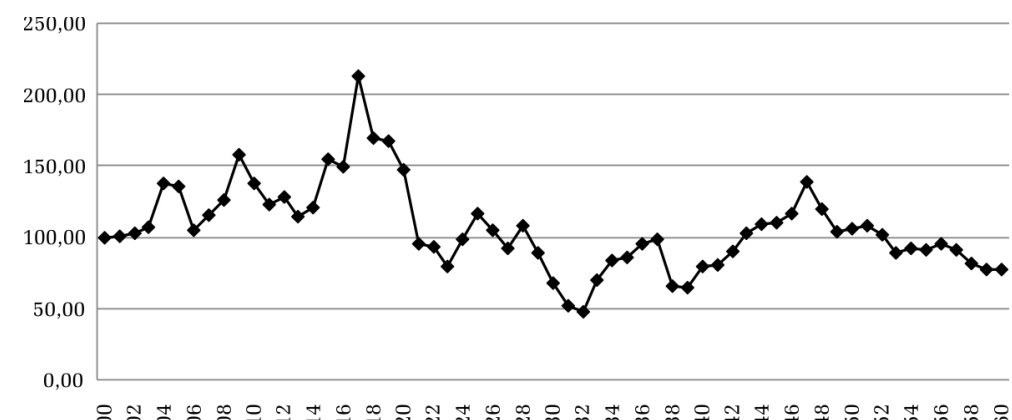

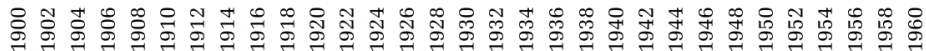
Fuente: David S. Jacks (2019). From Boom to Bust: A Typology of Real Commodity Prices in the Long Run. Diponible en https://www.nber.org/system/files/working_papers/w18874/w18874.pdf . Acceso en 29.10.2020 ón mundial, los precios de las materias primas se incrementaron consistentemente, lo cual permitió contar con recursos para impulsar el proyecto de nación del populismo, como puede observarse en la siguiente gráfica para el caso del trigo. da, a través del Estado, la transformación del régimen liberal al régimen de acumulación por sustitución de importaciones, que permitió poner a la industria en el centro y someter a su lógica de funcionamiento al resto de los sectores productivos.

Tales contradicciones permiten el surgimiento de los regímenes populistas, -antecedidos por movimientos sociales popularesque acceden al gobierno y también al poder, merced al debilitamiento de la oligarquía, así como a las alianzas de clases que establecen en este equilibrio inestable que constituyeron sus gobiernos.

Los gobiernos populistas emergieron en México con Lázaro Cárdenas del Río, en Argentina con Juan Domingo Perón, Getulio Vargas en Brasil, Rómulo Ernesto Betancourt en Venezuela, Jorge Eliécer Gaitán en Colombia, Paz Estenssoro en Bolivia, Carlos Ibáñez del Campo en Chile y Velasco Ibarra en Ecuador (Moira; Petrone, 1999, p. 20). El gran número de gobiernos muestra la generalización del fenómeno en la región, sustentado en condiciones favorables del mercado mundial.

A partir de 1932, pero sobre todo de 1939, con el inicio de la segunda conflagraci- 
Toda vez que la incipiente industria nacional producía para el mercado interno, el consumo de amplias masas obreras y campesinas era fundamental para su reproducción, por lo que la distribución del ingreso tenía el sentido de apuntalar la acumulación, con más énfasis que el criterio clientelar, que también existió pero subordinado al interés económico.

Los regímenes populistas tenían, como hemos señalado, una clara posición antiimperialista, lo cual fue un factor relevante para impulsar el desarrollo nacional centrado en la industria, bajo la concepción promovida por la Cepal. ${ }^{7}$ Por ello fue muy relevante la nacionalización de los recursos naturales, como el petróleo, los recursos mineros, la electricidad y el gas, para fortalecer la producción industrial y agrícola nacional.

En el ámbito rural el populismo trajo cambios estructurales de gran envergadura. Aun cuando la mayoría de los autores plantean que el populismo fue un fenómeno básicamente urbano (Ianni, 1975, p. 166), como en el caso de Argentina y Brasil, y aceptan que solo tuvo repercusiones rurales en México y Bolivia; en este ensayo planteamos que el populismo transformó radicalmente la relación industria-agricultura, sobre todo en los países grandes donde se desarrolló la industrialización, pues puso a la agricultura al servicio del sector manufacturero, hecho que no existía anteriormente.

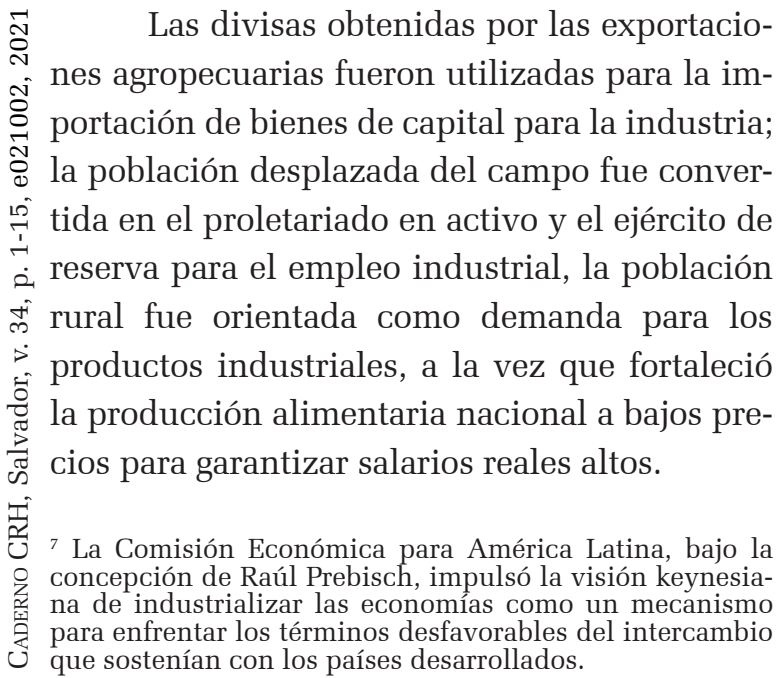

Además, en los casos de México con Cárdenas y Bolivia con Paz Estenssoro, se impulsó un cambio radical en el campo con la implementación de la reforma agraria. En el primer país, el impulso de los ejidos colectivos, el acto de armar a los campesinos para defender las tierras del embate de los terratenientes, la creación de Instituciones Estatales para impulsar la producción básica, etc., trajeron consigo la inclusión productiva de los campesinos, quienes tuvieron, a partir de entonces, un rol funcional en el proceso de acumulación.

Si bien los regímenes populistas impulsaron un proceso de corporativización de las clases subalternas, con lo cual se menguó su potencial de lucha, dichas clases tuvieron un papel fundamental en la transformación de los países y, sobre todo, alcanzaron el rol de incluidos en el régimen de acumulación, como productores y consumidores, pues, como señalamos, su producción y su demanda formaban parte de la reproducción del capital de punta.

\section{LA TRANSICIÓN HEGEMÓNICA DEL SIGLO XXI Y EL PROGRESISMO}

A partir del 2003, la fase de transición hegemónica iniciada en los años setenta ingresó en una crisis de hegemonía debido, entre otros, a dos procesos principales: el aumento tendencial de los precios del petróleo y las materias primas agrícolas y mineras, y el estallamiento de la segunda guerra de Irak, impulsada por Estados Unidos con el fin de apropiarse del petróleo de dicha nación asiática.

Ambos procesos expresaban el declive hegemónico de Estados Unidos, pues perdió el control sobre los precios del hidrocarburo e impulsó una guerra sin el consenso de las élites mundiales, que a la postre acabaría por perder, por lo que dicha potencia empezó a ejercer el dominio sin hegemonía (Arrighi, 2007, p. 160).

Además del declive hegemónico, la transición capitalista albergaba, como señala- 
mos, el agotamiento del régimen de acumulación neoliberal, que se expresó cabalmente en la crisis capitalista mundial iniciada en el año de 2008.

Las causas estructurales de dicha crisis remiten, en primer lugar, a la incapacidad del régimen de acumulación neoliberal para generar áreas de inversión productivas rentables, debido esencialmente a la imposición de elevadas cuotas de explotación que generaron altas tasas de plusvalía, pero también una restringida capacidad de consumo de la población. Tal hecho fue gestando, como en la crisis del 29, un proceso de sobreacumulación, que tuvo como contraparte el flujo del capital hacia el área financiera con el fin de valorizarse. Esta contradicción fortaleció el proceso de financiarización, mediante el cual el capital financiero domina al capital productivo y se desarrolla a expensas de él, al absorber valor sin reinvertirlo, lo cual lo convierte en un sector parasitario que tiende a atrofiar a la pequeña y mediana industria.

La sobreacumulación se tradujo, desde la perspectiva material, en un proceso de sobreproducción de mercancías sin posibilidades de realización o de venta. Esta situación fue resuelta creando una demanda ficticia, a través del impulso del endeudamiento entre la población, a quien se otorgaba crédito sin garantía real, con el fin de ampliar el mercado y crear poder de compra. La incapacidad del régimen de acumulación para generar una demanda creciente de capitales productivos y de mercancías, dado su carácter excluyente, derivó así en el crecimiento insospechado de capitales especulativos sin correspondencia con la creación de valor, y en mercancías vendidas a consumidores endeudados sin capacidad real de pago. Tal forma de funcionamiento puede desarrollarse durante una etapa expansiva del capital, pero tiende necesariamente a colapsarse ante la separación del valor de su representación dineraria, creando una burbuja que tarde o temprano acaba por estallar. Esto fue lo que ocurrió en 2008 en Estados Unidos, cuando los créditos otorgados sin respaldo en el campo inmobiliario no pudieron cobrarse, provocando una cadena de quiebras del capital involucrado.

En el ámbito latinoamericano, la crisis trajo consigo dos procesos esenciales. Por un lado, la revalorización de las materias primas agrícolas y mineras ${ }^{8} \mathrm{y}$, por otro, una mayor libertad política debido al declive hegemónico de Estados Unidos y su orientación hacia medio oriente. Estas condiciones permitieron, desde un punto de vista estructural, el surgimiento de gobiernos alternativos al neoliberalismo.

La crisis capitalista trajo también el debilitamiento de las fracciones financieras del capital dominante, así como del capital exportador, debido al declive de la demanda en los países desarrollados que enfrentaban la caída de la rentabilidad. En este contexto ocurrió de nuevo un vacío de poder, un equilibrio catastrófico de las élites que habían dominado durante el Neoliberalismo, pero fundamentalmente de sus representaciones políticas, que agotaron sus mecanismos de control, ante el descontento masivo de las clases populares orilladas a la exclusión y la marginalidad.

Así, al igual que en los años 30, emergieron gobiernos alternativos, como una corriente de transformación política y económica en América Latina, de corte antiimperialista y sostenida en el apoyo popular. En 1999 tomó posesión Hugo Chávez en Venezuela, en 2003 Lula en Brasil, Néstor Kirchner en Argentina también en 2003, Tabaré Vázquez en Uruguay en 2005, Evo Morales en Bolivia en 2006, Manuel Zelaya en Honduras en 2006, Rafael Correa en Ecuador en 2007, Cristina Kirchner que sucedió a su esposo en Argentina en 2007, Fernando Lugo en Paraguay en 2008, Mauricio Funes en El Salvador en 2009, la reelección de Evo Morales en 2009, el ascenso de José Múji${ }^{8} \mathrm{El}$ precio del petróleo se incrementó en $148.2 \%$ en julio de 2008 respecto a enero de 2007, el del arroz se incrementó en $222 \%$ de enero de 2007 a mayo de 2008, el del maíz en $73.9 \%$ de enero de 2007 a junio de 2008. En cuanto a los metales, el oro registró un incremento de $48.9 \%$ de enero de 2007 a julio de 2008, mientras que el cobre aumentó en $48.4 \%$ en el mismo periodo. Datos obtenidos de FMI STATS el 19 de marzo de 2021. 
ca que sucedió a Tabaré Vázquez en Uruguay en 2010, Dilma Rousseff que sucedió a Lula en Brasil en 2011, a lo que se suma la reelección de Cristina Kirchner en 2011, la de Rafael Correa en 2013, la elección de Maduro en Venezuela en 2013 y la reelección de Evo Morales en 2014; el regreso de Tabaré Vázquez en Uruguay en 2015, el ascenso de Andrés Manuel López Obrador en México, en 2018, de Alberto Fernández en Argentina en 2019 y de Luis Arce en Bolivia en 2020.

Estos gobiernos han tenido alcances y características muy diferentes, dependiendo de las condiciones históricas de cada país, los movimientos que los apoyaron, la forma en que llegaron al poder y las oligarquías que han enfrentado. Donde se desarrollaron procesos más radicales fue en los países andinos, Bolivia, Venezuela y Ecuador, mientras que Brasil, Argentina, Uruguay y México han seguido pautas más moderadas. A su vez, los pequeños países como El Salvador enfrentaron muchas dificultades para afianzarse. Sin embargo, el conjunto comparte una posición no alineada ante Estados Unidos, impulso al gasto social, la nacionalización de los recursos naturales y un rechazo implícito o explícito al Neoliberalismo.

De forma similar al populismo, los gobiernos progresistas impulsaron una política basada en ejes distintos al neoliberalismo. En primer lugar, expropiaron los recursos naturales con el fin de favorecer las finanzas estataఫิ les, dando aire así a un manejo más autónomo กิ del poder. Evo Morales decretó la nacionalización de los hidrocarburos, especialmente el ¿ gas en mayo del 2006, mientras que en octubre de ese año estatizó la mina de estaño Huanu$\therefore$ ni. En enero del 2009 nacionalizó la petrolera ले Chaco, mientras que en mayo del 2010 nacionalizó cuatro empresas eléctricas. En Venezuela Chávez nacionalizó en 200830 campos petroleros de la Faja del Orinoco, mientras que en I noviembre de este año nacionalizó la mina de oro "Las Cristinas". En marzo del 2009, a raíz de la crisis alimentaria, expropió las plantas procesadoras de arroz de Cargill. Este mismo año expropió dos centrales azucareras de capital colombiano, así como la Planta Compresora de Gas PIGAP II, de capital estadounidense. En 2010 expropió MONACA del grupo mexicano GRUMA y nacionalizó la empresa española Agroisleña, distribuidora de productos del campo, entre otras nacionalizaciones. En Ecuador, Rafael Correa hizo los trámites correspondientes para que el Estado ecuatoriano se apropiara del 100\% del crudo y el gas en el 2010. En Argentina, Cristina Kirchner expropió el 51\% de las acciones de Yacimientos Petrolíferos Fiscales (YPF) a la empresa española REPSOL el 3 de mayo del 2012; ese mismo año nacionalizó los Fondos de Pensiones y en 2015 nacionalizó los ferrocarriles de propiedad inglesa.

Asimismo, los países progresistas construyeron Instituciones como la Alianza Bolivariana de las Américas (Alba), PETROCARIBE, la Unión de Naciones del Sur (Unasur), y el Banco del Sur (Gauster; Fradejas, 2008, p. 43), que permitieron formar un bloque regional para enfrentar los embates de los países desarrollados, en particular de Estados Unidos, y apoyarse mutuamente ante situaciones de emergencia. Por otra parte, en el plano legislativo avanzaron, sobre todo los países andinos, en el establecimiento consensuado de una Nueva Constituyente que implicó la refundación de los Estados.

En el plano económico los países gobernados por el progresismo mostraron un desempeño superior al que registraron en la etapa neoliberal, así como al de aquellos países que continuaron con este tipo de políticas. Tal es la situación de Argentina quien a partir del gobierno de Néstor Kirchner, en 2003, registró tasas del PIB muy altas, de entre $6.8 \%$ y $9.2 \%$, mientras en los años del 2000 al 2002 registró tasas negativas. Bolivia, por su parte, registró tasas anuales de crecimiento del $6.1 \%$ en 2008 y $5.2 \%$ en 2011. Todavía hasta antes del golpe perpetrado en 2018, registraba una tasa de crecimiento del $4.6 \%$, superior al del resto de la región. Brasil registró tasas del $6.1 \%$ en 2007 y 7.5\% en 2010, cuando antes del gobierno de 
Lula solo había alcanzado 4.3\%. Venezuela llegó a crecer $18.4 \%$ en el 2004 y $10.3 \%$ en el 2005, mientras Ecuador registró un 8\% de crecimiento en el 2011. ${ }^{9}$

Una característica que comparten todos los gobiernos alternativos ha sido la disminución de la pobreza a niveles no vistos en la etapa reciente. De esta suerte, la pobreza se redujo en Brasil de 38.8\% de la población en 2003 a 19.9\% en 2017; mientras que en Bolivia el tránsito fue de $66.1 \%$ en el 2000 al $35.1 \%$ en 2017; en Ecuador pasó de $64.4 \%$ en el año 2000 a 22.8\% en 2017 (Cepal, 2019). En el caso de Argentina, el porcentaje de la población en pobreza pasó de 57,5\% en 2003 al 33.6\% en 2018 (BID, 2019; UCA, 2019). ${ }^{10}$

En el ámbito de lo rural, se observó un mayor énfasis en el apoyo agropecuario en relación a los gobiernos anteriores. Impulsaron políticas para dirigir la producción interna de alimentos, como en el caso de Venezuela, la Misión Mercal para controlar la distribución interna de alimentos a la vez que la Ley Orgánica de Seguridad y Soberanía Alimentarias. En Bolivia se impulsó la Empresa de Apoyo a la Producción de Alimentos (Empa) así como el Programa Productivo para la Seguridad Alimentaria en el 2008 y el Fondo para la Reconstrucción, Seguridad Alimentaria y Apoyo Productivo en la fase aguda de la crisis alimentaria. En Ecuador se emitió también la Ley Orgánica de la Seguridad Alimentaria en el 2009 y el Programa de Compras Públicas de Alimentos del Ministerio de Inclusión Económica y Social (Mies). En Brasil se impulsaron también las compras públicas a los pequeños productores rurales con el fin de crear una demanda y abastecer a escuelas, hospitales y cárceles.

Asimismo, durante las crisis alimentarias del 2008 y 2011, fueron los países no alineados los que mostraron un mejor desempeño como lo señala la FAO (2010, p. 48):

${ }^{9}$ Datos definitivos, obtenidos de CEPALSTATS el 4 de junio del 2013.

${ }^{10}$ Las cifras de pobreza corresponden al último dato disponible en cada caso.
En efecto, los países que han logrado amortiguar mejor las consecuencias de la crisis han sido aquellos donde el Estado tenía importante participación en áreas estratégicas, como el fomento integral a la producción de alimentos para el mercado interno, el financiamiento agrícola, el abastecimiento de alimentos (compras públicas, formación de reservas, distribución de alimentos) los programas de protección social, así como la capacidad de generar iniciativas para la diversificación de sus fuentes de importaciones de alimentos y la suscripción de acuerdos comerciales bilaterales e intrarregionales.

Por su parte, Rosero et al. (2011, p. 1112), señalan: "Los pocos países en desarrollo que han apostado a invertir en la agricultura, familiar como Brasil y Ecuador, han demostrado más capacidad para resistir el impacto de la crisis $[\ldots] "$.

A pesar de los avances logrados, sin embargo, podemos plantear que los gobiernos progresistas no lograron impulsar cambios que modificaran la estructura productiva, sustituyeran al capital dominante y permitieran la inclusión productiva de los campesinos e indígenas en el sistema de reproducción del capital.

En primer lugar, porque dejaron intacta la estructura agraria. En algunos países se hicieron intentos por modificar la tenencia de la tierra. Tal fue el caso de los países andinos, quienes desplegaron un conjunto de iniciativas referidas a la distribución de la tierra: Bolivia: Ley 3545 de Reconducción Comunitaria de la Reforma Agraria, básicamente de saneamiento de la propiedad; Venezuela: Ley de Tierras y Desarrollo Agrícola, autorizada por la Ley Habilitante en noviembre de 2001; y en Ecuador el Plan Nacional de Tierras en 2009.

Sin embargo, estas modificaciones no afectaron la propiedad privada. Las parcelas entregadas correspondieron al rescate de tierras ociosas, tierras que fueron apropiadas mediante procedimientos fraudulentos o irregulares, o que no cumplían con funciones sociales.

Por otra parte, los gobiernos aprovecharon el alza en los precios de las materias primas en el ámbito mundial, para apropiarse vía 
impuestos de la renta que generó la extracción de recursos naturales, fundamentalmente mineros y agrícolas por empresas transnacionales.

Los nuevos gobiernos progresistas han mantenido estrategias extractivistas. En los sectores clásicos, minero petrolero se persisten los principales rubros de producción y en algunos casos incluso se ha buscado ampliarlos y profundizarlos. Los ejemplos más destacados han sido la aprobación de una nueva norma de minería en Ecuador, la búsqueda de la explotación de hierro y litio en Bolivia y los primeros pasos de una mega explotación en Uruguay. De manera similar, estos gobiernos han promovido el sesgo extractivista de una agricultura basada en monocultivos de amplia cobertura geográfica y orientada a la exportación (Gudynas, 2010, p. 42).

Tal situación marca el origen de los gobiernos, pues fue precisamente el impulso de materias primas, como el oro, la plata, el cobre, los cultivos extractivos para agrocombustibles como el maíz, la palma africana, la caña de azúcar y los monocultivos para pastas oleaginosas como la soya, lo que les permitió lograr desde un principio la autonomía económica, por supuesto junto con el petróleo y el gas. Sin embargo, el pecado original del llamado "extractivismo" estriba en que se ha impulsado con capital transnacional a quien se le otorgan concesiones o se le brindan condiciones favorables para la inversión por parte de los gobiernos. De modo que, si por una parte se nacionalizan y expropian recursos naturales en favor de la nación, por otro lado se abre la $\vec{\delta}$ puerta al capital extranjero para la extracción ลิ de recursos que afectan los territorios de co\% munidades indígenas y poblaciones aledañas a las zonas de extracción.

Tal situación implicó una contradicción estructural del proyecto alternativo, en primer ஓं término porque significó continuar con la for-

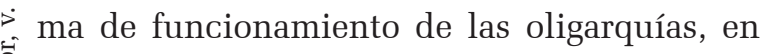
el sentido de depender del exterior y del capital transnacional para obtener los recursos. En segundo lugar porque dejó de lado el aspecto productivo interno, la industria nacional y la producción agrícola, como el motor fundamental de cambio, con lo cual se perdió la po- sibilidad de impulsar una transformación real. En tercer lugar, generó un conflicto permanente entre los gobiernos y las poblaciones afectadas por el avance del capital, el cual minó la fuerza ideológica del proyecto, pero, además, fracturó la alianza con las organizaciones indígenas y campesinas que pudieran darle fuerza al Estado ante las oligarquías que presionaron para su declive.

Toda vez que la transformación productiva no constituyó el eje central del proyecto alternativo, tuvo un papel destacado, en la política social, el asistencialismo (Borón, 2013; Estrada, 2012; Modonesi, 2019; Sader, 2008). Se impuso una tendencia de los gobiernos a transferir ingresos en efectivo a grupos vulnerables con programas como Bolsa Familia de Brasil, Bono de desarrollo Humano de Ecuador, Red Solidaria de El Salvador, Equidad en Uruguay, Programa Familias por la Inclusión Social en Argentina, Tekoporá en Paraguay, entre otros.

Podemos concluir que el proyecto alternativo al neoliberalismo avanzó en el terreno de la apropiación estatal de los recursos naturales estratégicos, construyó un polo contrahegemónico que enfrentó los embates del poder internacional, incrementó el gasto social, cambió la orientación regresiva del ingreso que caracterizó al neoliberalismo, fortaleció la agricultura al tiempo que impulsó la Constituyente; sin embargo, no logró afianzar un eje productivo interno por lo que surgió con "cola de paja” al fincarse en los proyectos extractivos transnacionalizados. Cuando los precios de las materias primas cayeron en 2014, ${ }^{11}$ se debilitó la fuente de los ingresos del progresismo, con lo cual, aunado a las contradicciones propias del proyecto, se abrió el flanco para la ofensiva de la derecha, que impulsó los golpes blandos en los casos de Honduras, Paraguay, Brasil y Bolivia, así como el ascenso electoral de líderes de

${ }^{11}$ El precio del petróleo registró una disminución de -65.3\% en diciembre de 2015 respecto a julio de 2013; el del maíz cayó un $-41.1 \%$ en diciembre de 2014 respecto a enero de 2013, mientras el del arroz cayó un -28.4\% de enero de 2013 a 2014. Por su parte el precio del oro se redujo en un $-28.2 \%$ de enero de 2013 a diciembre de 2014. Datos obtenidos de FMI STATS, el 19 de marzo de 2021. 
derecha como Macri en Argentina, Lenin Moreno en Ecuador y Bolsonaro en Brasil.

\section{UNA VISIÓN COMPARATIVA EN- TRE EL POPULISMO Y EL PRO- GRESISMO}

La pregunta central que rige este artículo consiste en indagar porque, al amparo del progresismo, no pudieron ocurrir cambios estructurales como durante el populismo.

En primer lugar, durante la transición del siglo XX, el populismo surgió cuando ya existían las condiciones para el ascenso de la clase alternativa que sustituyó a la oligarquía terrateniente, es decir, la burguesía industrial. Las condiciones para el ascenso del proceso de industrialización estaban ya maduras, en tanto la segunda guerra mundial impedía, en un plano internacional, obtener los bienes industriales requeridos por los países vía importaciones, por lo que era viable impulsarlos internamente. Como señalamos, el populismo constituye un régimen que alienta la industrialización ante la debilidad de la incipiente burguesía industrial y el declive de la clase terrateniente. ${ }^{12}$

Las condiciones maduras en lo económico y en la geopolítica mundial para el ascenso de un nuevo régimen de acumulación generaron, por tanto, una correlación de fuerzas favorable para la burguesía industrial naciente, lo cual potenció enormemente la capacidad transformadora del populismo.

En la fase de transición del siglo XXI, en cambio, no se encuentran maduras las condiciones para el ascenso de una clase alternativa al capital financiero y corporativo que son dominantes, toda vez que la burguesía industrial se encuentra debilitada por el proceso de de-

${ }^{12}$ La clase terrateniente se debilitó en el sentido de que dejó de ser la clase dominante en el poder, pues fue sustituida por la burguesía industrial. No obstante conservó un enorme poder que obligó a los gobiernos populistas a establecer alianzas con ella. Hasta la fecha sigue siendo un sector importante aunque ha experimentado cambios fundamentales al aliarse o someterse a sectores extranjeros para la explotación de los cultivos comodines. sindustrialización impulsado durante el neoliberalismo, a la vez que sectores importantes de ella se encuentran subordinados al capital corporativo y financiero. ${ }^{13}$ La crisis de 2008 trajo consigo el debilitamiento del sector financiero en el ámbito mundial, pero en la salida de la crisis los gobiernos de los países desarrollados erogaron inmensas sumas para rescatarlo, apuntalando así al sistema neoliberal vigente y postergando la caída definitiva de este sector (Rubio, 2017).

En América Latina se debilitó el capital financiero ante la crisis mundial, pero el sector corporativo cobró fuerza debido al aumento en los precios del petróleo y las materias primas. En este ámbito surgieron las condiciones para el ascenso del sector neoextractivista, como ya señalamos. En tal contexto, las divisas obtenidas por este sector resultaron providenciales para la obtención de ingresos de los gobiernos progresistas, sin embargo, el capital extractivista no constituye la alternativa al capital dominante del neoliberalismo. Es un sector atrasado que medra coyunturalmente de la situación internacional y no favorece el cambio a fondo de las estructuras productivas. Por esto mismo, no se puede plantear que el progresismo impulsa un modelo primario exportador emergente, pues los precios elevados fueron coyunturales (2003-2014). Estos gobiernos, en cambio, forman parte de una transición capitalista sin resolución de la crisis estructural del capitalismo. Lo paradójico de la situación es que, mientras el populismo derrotó a la oligarquía exportadora de bienes primarios, una clase atrasada y retrógrada para sustituirla por una burguesía industrial de avanzada, casi cien años después el progresismo se apoyó en un sector muy similar a la oligarquía, aunque con un carácter corporativo transnacional, pero también exportador de bienes primarios y dependiente de la coyuntura internacional, sin

13 Cabe señalar también que para impulsar al capital industrial en la actual situación, se requiere enfrentar al capital financiero que medra del estancamiento productivo. Sin embargo, los gobiernos progresistas no han tenido la fuerza suficiente para lograrlo. 
tener la posibilidad de sustituirlo por un sector capitalista de avanzada.

Mientras en el populismo, además del dominio inglés, se debilitó también la clase dominante encarnada por la oligarquía exportadora, en cambio en el Progresismo lo que se debilitó fueron los mecanismos internacionales de dominio de Estados Unidos sobre los países latinoamericanos, debido a la crisis de 2008 y a la derrota en la guerra de Irak, como ya señalamos, sin que ello condujera a un debilitamiento real de las élites nativas. En cambio, este declive del dominio imperial sí repercutió en un debilitamiento de las representaciones políticas de las élites en poder, ante el hartazgo de las clases subalternas golpeadas por la crisis económica y alimentaria.

En este proceso no emergió aún la clase de la burguesía que sustituirá al capital financiero, por lo que los gobiernos progresistas se vieron imposibilitados para abanderar esta transformación. Lo cual quiere decir que las condiciones económicas y sociales de la transición no estaban aún maduras para el impulso de un nuevo régimen de acumulación que sustituyera al neoliberalismo. En este contexto, el progresismo surge prematuramente, ante una fase de transición que se prolonga enormemente sin poder dar a luz a la nueva configuración del capital.

Tanto en el populismo como en el progresismo hay por tanto un vacío de poder, pero ㅊ en el populismo viene por el debilitamiento i del dominio inglés y de la oligarquía terrateniente, mientras en el progresismo viene del debilitamiento en el ámbito mundial del capital financiero y de la hegemonía norteamerica$\therefore$ na, sin que se concrete en el debilitamiento del fi capital financiero y corporativo local.

Mientras en el Populismo los gobiernos y las clases populares enfrentaron como su enemigo principal a la oligarquía terrateniente, en el Progresismo, ante la ausencia de una clase de avanzada, los gobiernos se vieron obligados a aliarse con el capital extractivista ante el embate del capital (golpe de estado en
Venezuela y motín de los policías en Ecuador, contienda separatista de Santa Cruz en Bolivia, movilizaciones de la derecha en Brasil, lucha contra las retenciones en Argentina), lo cual evidencia que la correlación de fuerzas del progresismo no fue tan favorable como en el caso del populismo. Tal situación trajo consigo que el Progresismo no cuente con un proyecto alternativo de transformación estructural en los países donde se instala.

Desde la perspectiva de las políticas sociales, durante el populismo las políticas distributivas tenían el sentido de impulsar la demanda popular para apuntalar a la industria, como señalamos. Resultaba fundamental mantener bajos los precios de los alimentos y altos los salarios reales para fortalecer el consumo popular en beneficio de la industria. En cambio, en el progresismo, las políticas distributivas han caído en el vacío, pues no apuntalan a un nuevo régimen de acumulación, y por lo tanto, como señalamos, se han reducido a un nivel asistencialista para fortalecer su base social de sustentación. Los recursos fueron distribuidos para atemperar la pobreza, sin tener el objetivo de incrementar el consumo, pues los sectores productivos de punta se orientan a la exportación.

Mientras el Populismo se apoya en clases sociales esenciales del capital como los obreros y los campesinos, en el progresismo las clases subalternas beneficiadas son sectores populares marginales, a quienes se destina prioritariamente el gasto asistencialista. Si bien existen políticas de mejoramiento de los salarios y las condiciones laborales de los obreros, son de menor importancia, en tanto dicho mejoramiento no contribuye a impulsar la producción y el consumo interno y, por tanto, a un sector del capital de avanzada.

En el ámbito rural, durante el populismo se reorganizó la relación industria-agricultura, poniendo ésta última al servicio del sector manufacturero, como ya señalamos. En este contexto, el impulso a la producción alimentaria a bajos precios tenía el sentido de apuntalar la acumulación elevando los salarios reales al 
mismo tiempo que la explotación de la fuerza de trabajo. Por ello fue tan importante la reforma agraria en México y Bolivia, que permitió distribuir la tierra a los campesinos, capaces de garantizar bajos precios al ahorrar la renta de la tierra. Por esta razón se logró la inclusión productiva de los campesinos en los países donde eran importantes numéricamente.

En cambio, durante el progresismo, persistió el dominio corporativo sobre el sector rural, apuntalado por el aumento en los precios de las materias primas, con lo cual los intentos de transformación del campo fueron limitados, en tanto no ha surgido un nuevo régimen de acumulación que ubique el rol funcional de la agricultura y los campesinos en términos productivos y ambientales.

Continuó el mecanismo de la importación abaratada de granos básicos proveniente de los Estados Unidos, mediante los acuerdos comerciales, para satisfacer la demanda a bajos costos de las grandes transnacionales, con lo cual la producción alimentaria de los pequeños productores siguió siendo marginal. Aun cuando se impulsaron programas de compras públicas para generar demanda a los productores, en los casos de Brasil y Ecuador, y de precios de garantía, en el caso de México, no dejaron de tener un rol marginal, pues la alimentación básica de la población recayó en las importaciones o bien en las grandes corporaciones agroalimentarias, con lo cual persistió la exclusión productiva de los campesinos.

Tanto durante el populismo como en el progresismo existieron precios altos de las materias primas en el ámbito mundial, lo cual les permitió contar con recursos para impulsar las políticas sociales, pero estas fueron de distinto contenido. En el populismo los precios elevados provenían de la segunda guerra mundial, mientras que en el progresismo fueron resultado de la crisis alimentaria. En el populismo la revalorización de las materias primas permitió impulsar la industrialización, mientras en el progresismo alentó el neoextractivismo, que sometió a los países a una mayor dependencia con el mercado mundial, a la vez que fracturó su base social, al impactar los recursos naturales de las comunidades campesinas e indígenas.

Como es sabido, los gobiernos progresistas de Evo Morales y Rafael Correa enfrentaron la oposición de los movimientos indígenas a los proyectos de la carretera que afectaba al parque TIPNIS en Bolivia y la explotación petrolera del bloque 79 en Ecuador, que afectaba a los territorios de las nacionalidades indígenas sapara y kichwa. Tal es el caso también de la oposición indígena actual del Ejército Zapatista de Liberación Nacional (EZLN) y otras organizaciones indígenas contra la construcción del Tren Maya, que impulsa en México el presidente Andrés Manuel López Obrador.

Podemos concluir, entonces, que las condiciones económicas nativas y mundiales del populismo fueron favorables a la transformación e inclusión de las clases subalternas, mientras que en el progresismo las condiciones han sido adversas, por lo que los cambios han sido superficiales, de tal manera que, cuando dichos gobiernos son abatidos por la derecha, esta puede echar por la borda las transformaciones logradas.

Aun cuando los gobiernos progresistas no afectaron básicamente los intereses de la burguesía, el capital transnacional tiende a eliminarlos con los golpes blandos y la "democracia" mediática, porque se requieren gobiernos autoritarios para elevar la cuota de explotación con el fin de salir de la crisis capitalista. Los gobiernos progresistas son por ello un obstáculo al capital transnacional.

Toda vez que el fenómeno del progresismo no ha concluido, pues al momento de escribir este artículo siguen surgiendo gobiernos de esta índole en América Latina, consideramos, a manera de hipótesis, que conforme avance la transición capitalista y logren dominar los elementos emergentes sobre los decadentes, es decir, en la medida en que maduren las condiciones para una transformación hegemónica y del régimen de acumulación, la capacidad transformadora de los gobiernos progresistas podrá 
ser mayor. Para eso, sin embargo, falta todavía vencer las posiciones neofascistas de la derecha que se encuentran en ascenso. Por ello, el papel de los movimientos populares es fundamental en la transición. Ahí está la esperanza.

Recebido para publicação em 10 de novembro de 2020 Aceito em 05 de abril de 2021

\section{REFERENCIAS}

ARRIGHI, G. Adam Smith en Pekín: orígenes y fundamentos del siglo XXI. Madrid: Akal, 2007.

BORÓN, A. ¿Una nueva era populista en América Latina? In: VÁSQUEZ, G. H. (org.). El eterno retorno del populismo en América Latina y el Caribe. Bogotá: Pontificia Universidad Javeriana, 2012. p. 131-158.

BORÓN, A. América Latina en la geopolítica del imperialismo. Buenos Aires: Luxemburg, 3. ed., 2013.

BID. Estadísticas de pobreza y desigualdad. BID, Washington, 2019. Disponible en: https://www.iadb.org/ es/investigacion-y-datos/pobreza\%2C7526.html. Acceso en: 27 out. 2020.

CARDOSO, F.; FALETTO, E. Dependencia y desarrollo en América Latina: ensayo de interpretación sociológica. México: Siglo XXI Editores, 1969.

CEPAL. Estadísticas e Indicadores Sociales: pobreza. Santiago de Chile: Cepal, 2019. Disponible en: https://cepalstatprod.cepal.org/cepalstat/tabulador/ConsultaIntegrada. asp?IdAplicacion $=1 \&$ idTema $=927 \&$ idIndicador $=3335 \&$ idioma $=$ e. Acceso en: 27 out. 2020 .

DUSSEL, E. Cinco tesis sobre el populismo. In: MÁRQUEZ, PASTRANA y HOYOS (org.). El eterno retorno del populismo en América Latina y el Caribe, Bogotá: Pontificia Universidad Javeriana - Clacso, 2012. p. 159-179.

ESTRADA, J. Elementos de economía política de la política social en América Latina. Reflexiones a propósito de los gobiernos progresistas. In: ESTRADA (org.). La crisis capitalista mundial y América Latina. Lecturas de Economía Política. Buenos Aires: Clacso, 2012. p. 311-329.

- FAO. Panorama de la seguridad alimentaria en América ऽิ Latina y el Caribe. Roma: FAO, 2010.

FMI. Primary Comodity Prices. FMI Stats. Washington, ○े 2021. Disponible en https://www.imf.org/en/Research/ ᄀ commodity-prices. Acceso en 19 mar. 2021.

GAUSTER, S.; FRADEJAS, A. Propuesta de is institucionalidad y políticas públicas para la promoción I de la agricultura campesina de Guatemala. Guatemala: $\therefore$ Instituto de Estudios Agrarios y Rurales, 2008.

Æீ GRAMSCI, A. Cuadernos de la cárcel. México: ERA, 1981.

$>$ GUDYNAS, E. Agropecuaria y nuevo extractivismo bajo ô los gobiernos progresistas de América del Sur. Revista Territorios, São Paulo, n. 5, p. 37-54, 2010.

Debates sobre el desarrollo y sus alternativas en América Latina: una breve guía heterodoxa. In: LANG, II M.; MOKRANI, D. (org.). Más allá del desarrollo. México: J Fundación Rosa Luxemburg, 2012. p. 21-53.

IANNI, O. La formación del Estado populista en América Latina. México: ERA, 1975

KATZ, C. Las disyuntivas de la izquierda en América
Latina. Buenos Aires: Luxemburg, 2008.

Miradas posdesarrollistas. Revista Herramienta, n. 62, 2015. Disponible en: https://herramienta.com.ar/ articulo.php?id=2303. Acceso en: 17 mar. 2021.

KAY, C.; VERGARA, L. La cuestión agraria y los gobiernos de izquierda en América Latina: campesinos, agronegocio y desarrollismo. Buenos Aires: Clacso, 2018.

KINDLEBERGER, C. La crisis económica: 1929-1939. Madrid: Traficantes de Sueños, 2009.

MODONESI, M. Revoluciones pasivas en América Latina: una aproximación gramsciana a la caracterización de los gobiernos progresistas de inicio del siglo. In: THWAITES, M. (org.). El Estado en América Latina: continuidades y rupturas. Santiago de Chile: Clacso, 2012. p. 139-166.

Revoluciones pasivas en América. México: UAMAzcapotzalco, 2017.

MODONESI, M. El progresismo latinoamericano: un debate de época. In: GAUDICHAUD, F.; WEBBER, J.; MODONESI, M. Los gobiernos progresistas latinoamericanos del siglo XXI: ensayos de interpretación histórica. México: FCPyS UNAM, 2019. p. 181-229.

MOIRA, M.; PETRONE, M. Los complejos de la cenicienta. In: MOIRA, M; PETRONE, M. (org.). Populismo y neopopulismo en América Latina: el problema de la cenicienta. Buenos Aires: Editorial Universitaria de Buenos Aires, 1999. p. 11-55.

MOUZELIS, N. Populismo y clientelismo como modos de incorporación de las masas en sistemas políticos semiperiféricos. In: VILAS, C. M. (org.). La democratización fundamental: el populismo en América Latina. México: Conaculta, 1994. p. 458-480.

OUVIÑA, H.; THWAITES, M. (org.). Estados en disputa: auge y fractura del ciclo de impugnación al neoliberalismo en América Latina. Buenos Aires: Bajo Tierra, 2018.

PIPITONE, U. La esperanza y el delirio: una historia de la izquierda en América Latina. México: Cide, 2015.

PIVA, A. ¿Cuánto hay de nuevo y cuánto de populismo en el neopopulismo? Kirchnerismo y peronismo en la Argentina post 2001. Trabajo y Sociedad, Santiago del Estero, n. 21, p. 135-157, 2013.

ROSERO, F. et al. Hacia nuevas políticas alimentarias en América Latina y Europa. Ecuador: Friedrich Ebert Srtiftung, 2011.

RUBIO, B. El dominio del hambre: crisis de hegemonía y alimentos. Ecuador: La Tierra, 2017.

SADER, E. Refundar el Estado: posneoliberalismo en América Latina. Buenos Aires: Clacso, 2008.

El nuevo topo: los caminos de la izquierda latinoamericana. Argentina: Siglo XXI, 2009.

SVAMPA, M. Debates latinoamericanos: indianismo, desarrollo, dependencia, populismo. Lima: Edhasa, 2018a.

Las fronteras del neoextractivismo en América $\overline{\text { Latina: }}$ conflictos socioambientales, giro ecoterritorial y nuevas dependencias. Guadalajara: Calas, 2018b.

UCA. Observatorio de la Deuda Social Argentina. Argentina: Pontificia Universidad Católica de Argentina, 2019. Disponible en: http://uca.edu.ar/es/observatorio-dela-deuda-social-argentina. Acceso en: 27 out. 2020.

VILAS, C. Estudio preliminar: el populismo o la democratización fundamental en América Latina. In: VILAS, C. (org.). La democratización fundamental: el populismo en América Latina. México: Conaculta, 1994. p. 11-118.

La izquierda latinoamericana y el surgimiento de regímenes nacional-populares. Buenos Aires: Nueva Sociedad, 2005 
FROM POPULISM TO PROGRESSISM: reflections on its transforming capacity

\author{
Blanca Rubio \\ Jaime Peña
}

The aim of the article is to analyze and contrast the populism of the 1930s with the progressivism of the 2000s, in Latin America, with an emphasis on rural areas. It is about investigating what were the conditions that allowed the transformative potential of populism and what were those that generated the recent phenomenon, which castrated its transforming capacity. The main argument points to the historical conditions in which both phenomena arose. While populism emerged when the conditions already existed to replace the landowning oligarchy with the industrial bourgeoisie and promote the accumulation regime by import substitution, progressivism emerged prematurely, when the decline of the neoliberal regime had begun but has not yet weakened. the power of financial and corporate capital, nor is the replacement of this class by another dominant sector in sight. This leads to an inability of progressivism to promote structural changes.

KEYwords: Hegemonic Transition. Populism. Progressivism. Hegemony.

\section{DU POPULISME AU PROGRESSISME: réflexions sur sa capacité de transformation}

\author{
Blanca Rubio \\ Jaime Peña
}

Le but de l'article est d'analyser et de confronter le populisme des années 30 au progressisme des années 2000, en mettant l'accent sur les zones rurales. Il s'agit de rechercher quelles étaient les conditions qui ont permis le potentiel transformateur du populisme et quelles ont été celles qui ont généré le phénomène récent, qui a castré sa capacité de transformation. Largument principal indique les conditions historiques dans lesquelles les deux phénomènes se sont produits. Alors que le populisme est apparu alors que les conditions existaient déjà pour remplacer l'oligarchie foncière par la bourgeoisie industrielle et promouvoir le régime d'accumulation par substitution aux importations, le progressisme est apparu prématurément, lorsque le déclin du régime néolibéral avait commencé mais ne s'est pas encore affaibli. Le pouvoir du capital financier et corporatif, ni le remplacement de ladite classe par un autre secteur dominant en vue. Cela conduit à une incapacité du progressisme à promouvoir des changements structurels.

Motsclés: Transition Hégémonique. Populisme. Progressisme. Hégémonie.

Blanca Rubio - Doctora en Economía por la UNAM. Investigadora Titular del Instituto de Investigaciones Sociales de la Universidad Nacional Autónoma de México. Entre sus publicaciones se encuentran los libros Explotados y excluidos. Los campesinos latinoamericanos en la fase agroexportadora neoliberal, El dominio del hambre. Crisis de hegemonía y alimentos y América Latina en la mirada. Las transformaciones rurales en la transición capitalista.

Jaime Peña - Doctor en Sociología por la UAM-Azcapotzalco y en Pedagogía por la UNAM. Investigador Titular de la Facultad de Estudios Superiores Acatlán, UNAM Entre sus publicaciones se encuentran los libros Crisis del agua en México, Agricultura y medio ambiente fronterizos, El agua, espejo de los pueblos y Nudos didácticos de la investigación multidisciplinaria. 
